\title{
Reducing the polynomial-like iterative equations order and a generalized Zoltán Boros' problem
}

\author{
Szymon Draga, Janusz MorawieC
}

\begin{abstract}
We present a technique for reducing the order of polynomial-like iterative equations; in particular, we answer a question asked by Wenmeng Zhang and Weinian Zhang. Our method involves the asymptotic behaviour of the sequence of consecutive iterates of the unknown function at a given point. As an application we solve a generalized problem of Zoltán Boros posed during the 50th ISFE.
\end{abstract}

Mathematics Subject Classification. 39B12, 39A06.

Keywords. Continuous solution, polynomial-like equation, characteristic equation, iterate, recurrence relation.

\section{Introduction}

Suppose $I \subset \mathbb{R}$ is a non-degenerated interval and let $g: I \rightarrow I$ be a function. Assuming $g$ to be continuous we are interested in lowering the order of the equation

$$
a_{N} g^{N}(x)+\cdots+a_{1} g(x)+a_{0} x=0,
$$

where $N$ is a positive integer, the coefficients $a_{0}, a_{1}, \ldots, a_{N}$ are real and $a_{0} \neq 0$; here and throughout this paper $g^{n}$ stands for the $n$-th iterate of $g$. Note that $a_{0} \neq 0$ implies that $a_{n} \neq 0$ for some $n=1, \ldots, N$. From now on we assume that $a_{N} \neq 0$. It turns out that continuous solutions to (1.1) deeply depend on the roots of its characteristic equation

$$
a_{N} r^{N}+\cdots+a_{1} r+a_{0}=0,
$$

which is usually obtained by assuming that $g$ has the form $g(x)=r x$. Up to now the case $N=2$ is the only non-trivial one which has been completely solved (see [13]). In fact, the problem still remains open even for $N=3$ (see $[7])$. These difficulties follow from the non-linearity of the operator $g \mapsto g^{n}$. Nonetheless, a lot of investigation was done in this matter; see a survey on 
functional equations with superpositions of the unknown function [1, Section 3] and a survey on iterative equations of polynomial type [20].

One of methods for finding solutions to Eq. (1.1), and also to its nonhomogenous counterpart, where zero on the right-hand side is replaced by an arbitrary continuous function, is based on lowering its order. The first such results on the whole real line were obtained in [8] in the case where all roots of the characteristic equation are real and satisfy some special conditions. Further research in this direction was done in $[16,18,21]$, but still most cases remain unsolved. For some exploration of Eq. (1.1) on intervals see $[6,11,12,14,17,19]$ and on half-lines see [4].

Let us note that (1.2) can also be considered as the characteristic equation of the recurrence relation

$$
a_{N} x_{m+N}+\cdots+a_{1} x_{m+1}+a_{0} x_{m}=0
$$

which might be obtained by choosing $x_{0} \in I$ arbitrarily and putting $x_{m}=$ $g\left(x_{m-1}\right)$ for all $m \in \mathbb{N}$. Such an approach we will examine in the present paper.

It can be easily observed that if a polynomial $b_{M} r^{M}+\cdots+b_{1} r+b_{0}$ divides a polynomial $a_{N} r^{N}+\cdots+a_{1} r+a_{0}$ and a function $g$ satisfies the equation

$$
b_{M} g^{M}(x)+\cdots+b_{1} g(x)+b_{0} x=0,
$$

then it satisfies also (1.1); for a simple proof see [9]. The main objective of this paper is to give particular conditions under which a converse holds, i.e., we want to find some conditions guaranteeing that if a continuous function $g$ satisfies (1.1), then there is a divisor of $a_{N} r^{N}+\cdots+a_{1} r+a_{0}$ such that $g$ is a solution to the corresponding iterative equation of lower order. Of course, it is not possible in each case. For instance, we may consider the equation $g^{2}(x)-2 g(x)+x=0$ whose continuous solution (on the whole real line) is $g(x)=x+c$, where $c$ is a constant.

The paper is organized as follows. Sect. 2 contains basic properties of solutions to the considered equations and preliminary information on linear homogenous recurrence relations. In Sect. 3 we prove theorems on eliminating non-real roots from the characteristic equation which show that, in new crucial cases, Eq. (1.1) is equivalent to an equation of lower order. In particular, we generalize results from [21] and give an answer to the question posed in the last section of that paper. In Sect. 4 we obtain similar results as in the previous section, but we eliminate real roots of opposite sign. These theorems allow us to solve, in Sect. 5, a generalized problem of Zoltán Boros. Namely, for a given integer $n \geq 3$ we determine all continuous self-mapping functions $f$ acting on an interval and satisfying

$$
f^{n}(x)=\frac{[f(x)]^{n}}{x^{n-1}} .
$$


For $n=2$ the equation above can be solved using the mentioned result of Nabeya or [10]. In the last section we discuss further problems.

\section{Preliminaries}

As we mentioned before we assume that $a_{0} \neq 0$.

Lemma 2.1. If a function $g$ satisfies (1.1), then it is injective.

Proof. Choose $x, y \in I$ and suppose that $g(x)=g(y)$. Then

$$
x=-\frac{1}{a_{0}}\left(a_{N} g^{N}(x)+\cdots+a_{1} g(x)\right)=-\frac{1}{a_{0}}\left(a_{N} g^{N}(y)+\cdots+a_{1} g(y)\right)=y .
$$

The foregoing lemma directly implies that every continuous solution to (1.1) is strictly monotone. It means that the sequence $\left(x_{m}\right)_{m \in \mathbb{N}_{0}}$ given by $x_{0} \in I$ and $x_{m}=g\left(x_{m-1}\right)$ for all $m \in \mathbb{N}$ is either monotone (in the case of increasing $g$ ) or anti-monotone (in the case of decreasing $g$ ). By anti-monotone we mean that the expression $(-1)^{m}\left(x_{m}-x_{m-1}\right)$ does not change its sign when $m$ runs through $\mathbb{N}_{0}$.

In the case where $g$ is bijective, $h=g^{-1}$ is also a self-mapping function acting on $I$. Putting $g^{-N}(x)$ in place of $x$ in (1.1) we obtain the equation

$$
a_{0} h^{N}(x)+\cdots+a_{N-1} h(x)+a_{N} x=0
$$

which is called the dual equation. It is worth mentioning that if $r_{1}, \ldots, r_{N}$ are roots of (1.2), then the roots of the characteristic equation of (2.1) are $r_{1}^{-1}, \ldots, r_{N}^{-1}$. Whence if a bijective function $g$ satisfies (1.1) and its inverse satisfies an iterative equation of a lower order, then the order of (1.1) can be reduced. Moreover, a root from the characteristic equation can be eliminated if and only if its inverse can be eliminated from the characteristic equation of the dual equation.

Lemma 2.2. A function $g$ satisfies Eq. (1.1) if and only if for every $x_{0} \in I$ the sequence $\left(x_{m}\right)_{m \in \mathbb{N}_{0}}$ given by $x_{m}=g\left(x_{m-1}\right)$ for all $m \in \mathbb{N}$ satisfies the recurrence relation (1.3).

For the theory of linear recurrence relations see, for instance, [5, §3.2]. We will recall only the most significant theorem in this matter. In order to do this and simplify the writing we introduce the following notation. For a given polynomial $c_{K} r^{K}+\cdots+c_{1} r+c_{0}$ we denote by $\mathcal{R}\left(c_{K}, \ldots, c_{0}\right)$ the set $\left\{\left(r_{1}, k_{1}\right), \ldots,\left(r_{p}, k_{p}\right)\right\}$ of all pairs of pairwise distinct roots $r_{1}, \ldots, r_{p}$ and their multiplicities $k_{1}, \ldots, k_{p}$, respectively. Here and throughout the paper by a polynomial we mean a polynomial with real coefficients. Note that in the introduced notation $k_{1}+\cdots+k_{p}$ equals the degree of $c_{K} r^{K}+\cdots+c_{1} r+c_{0}$. Moreover, $(\mu, k),(\bar{\mu}, k) \in \mathcal{R}\left(c_{K}, \ldots, c_{0}\right)$ forces $\mu$ to be non-real. 
Theorem 2.3. Assume

$$
\mathcal{R}\left(a_{N}, \ldots, a_{0}\right)=\left\{\left(\lambda_{1}, l_{1}\right) \ldots,\left(\lambda_{p}, l_{p}\right),\left(\mu_{1}, k_{1}\right),\left(\overline{\mu_{1}}, k_{1}\right), \ldots,\left(\mu_{q}, k_{q}\right),\left(\overline{\mu_{q}}, k_{q}\right)\right\} .
$$

Then a real-valued sequence $\left(x_{m}\right)_{m \in \mathbb{N}_{0}}$ is a solution to (1.3) if and only if it is given by

$$
x_{m}=\sum_{k=1}^{p} A_{k}(m) \lambda_{k}^{m}+\sum_{j=1}^{q}\left(B_{j}(m) \cos m \phi_{j}+C_{j}(m) \sin m \phi_{j}\right)\left|\mu_{j}\right|^{m}
$$

for all $m \in \mathbb{N}_{0}$, where $A_{k}$ is a polynomial whose degree equals at most $l_{k}-1$ for $k=1, \ldots, p$ and $B_{j}, C_{j}$ are polynomials whose degrees equal at most $k_{j}-1$, with $\phi_{j}$ being an argument of $\mu_{j}$, for $j=1, \ldots, q$.

\section{Eliminating non-real roots}

We will make use of the following lemma whose proof can be found in [15].

Lemma 3.1. Let the sequence $\left(x_{m}\right)_{m \in \mathbb{N}_{0}}$ be given by $x_{m}=\sum_{n=1}^{N}\left(a_{n} \cos m \phi_{n}+\right.$ $\left.b_{n} \sin m \phi_{n}\right)$ for all $m \in \mathbb{N}_{0}$ with $a_{n}, b_{n} \in \mathbb{R}$ and $\phi_{n} \in(0,2 \pi)$ for $n=1, \ldots, N$. If $\liminf \operatorname{in}_{m \rightarrow \infty} x_{m} \geq 0$, then $x_{m}=0$ for all $m \in \mathbb{N}_{0}$.

Lemma 3.2. Suppose $\lambda_{1}, \ldots, \lambda_{N}$ to be complex numbers such that $\lambda_{n}=$ $r\left(\cos \phi_{n}+\mathrm{i} \sin \phi_{n}\right)$ with $r>0$ and $\phi_{n} \in(0, \pi)$ for $n=1, \ldots, N$. Moreover, let $F: \mathbb{N}_{0} \rightarrow \mathbb{R}$ be a function such that $\lim _{m \rightarrow \infty} F(m) / r^{m}=0$ and let $A_{n}, B_{n}$ be polynomials for $n=1, \ldots, N$. If the sequence $\left(x_{m}\right)_{m \in \mathbb{N}_{0}}$ given by

$$
x_{m}=F(m)+\sum_{n=1}^{N} r^{m}\left(A_{n}(m) \cos m \phi_{n}+B_{n}(m) \sin m \phi_{n}\right) \quad \text { for all } m \in \mathbb{N}_{0}
$$

is either monotone or anti-monotone, then $x_{m}=F(m)$ for all $m \in \mathbb{N}_{0}$.

Proof. Assume that $k$ is the greatest number from the degrees of polynomials $A_{1}, \ldots, A_{N}$ and $B_{1}, \ldots, B_{N}$. We may write $A_{n}(m)=\sum_{l=0}^{k} a_{l}^{(n)} m^{l}$ and $B_{n}(m)=\sum_{l=0}^{k} b_{l}^{(n)} m^{l}$ for $n=1, \ldots, N$, where some of the coefficients $a_{k}^{(n)}$ 's and $b_{k}^{(n)}$ 's are possibly zeros.

We first consider the case where $\left(x_{m}\right)_{m \in \mathbb{N}_{0}}$ is monotone. By considering the sequence $\left(-x_{m}\right)_{m \in \mathbb{N}_{0}}$ if necessary, we may assume that $x_{m} \geq 0$ for all but finitely many $m \in \mathbb{N}_{0}$. It means that the inequality

$$
\frac{F(m)}{r^{m} \cdot m^{k}}+\sum_{n=1}^{N}\left(\frac{A_{n}(m)}{m^{k}} \cos m \phi_{n}+\frac{B_{n}(m)}{m^{k}} \sin m \phi_{n}\right) \geq 0
$$

holds for all but finitely many $m \in \mathbb{N}$. Consequently, we obtain

$$
\liminf _{m \rightarrow \infty} \sum_{n=1}^{N}\left(\frac{A_{n}(m)}{m^{k}} \cos m \phi_{n}+\frac{B_{n}(m)}{m^{k}} \sin m \phi_{n}\right) \geq 0
$$


and hence

$$
\liminf _{m \rightarrow \infty} \sum_{n=1}^{N}\left(a_{k}^{(n)} \cos m \phi_{n}+b_{k}^{(n)} \sin m \phi_{n}\right) \geq 0 .
$$

In view of Lemma 3.1

$$
\sum_{n=1}^{N}\left(a_{k}^{(n)} \cos m \phi_{n}+b_{k}^{(n)} \sin m \phi_{n}\right)=0 \quad \text { for all } m \in \mathbb{N}_{0},
$$

which obviously implies

$$
\sum_{n=1}^{N}\left(a_{k}^{(n)} m^{k} \cos m \phi_{n}+b_{k}^{(n)} m^{k} \sin m \phi_{n}\right)=0 \quad \text { for all } m \in \mathbb{N}_{0}
$$

It means that we can eliminate terms with $m^{k}$ from $\sum_{n=1}^{N}\left(A_{n}(m) \cos m \phi_{n}+\right.$ $\left.B_{n}(m) \sin m \phi_{n}\right)$ and now we can assume that the greatest number from the degrees of polynomials $A_{n}$ and $B_{n}$ is equal to $l \leq k-1$. The same procedure shows that the terms with $m^{l}$ can also be eliminated. Continuing in this fashion, we obtain

$$
\sum_{n=1}^{N}\left(A_{n}(m) \cos m \phi_{n}+B_{n}(m) \sin m \phi_{n}\right)=0 \quad \text { for all } m \in \mathbb{N}_{0}
$$

Secondly, we consider the case where $\left(x_{m}\right)_{m \in \mathbb{N}_{0}}$ is anti-monotone. By considering the sequence $\left(-x_{m}\right)_{m \in \mathbb{N}_{0}}$ if necessary, we may assume that we have $(-1)^{m}\left(x_{m}-x_{m-1}\right) \geq 0$ for all $m \in \mathbb{N}$. It implies that the inequality

$$
\begin{aligned}
& \frac{F(2 m)-F(2 m-1)}{r^{2 m} \cdot(2 m)^{k}}+\sum_{n=1}^{N}\left(\frac{A_{n}(2 m)}{(2 m)^{k}} \cos 2 m \phi_{n}+\frac{B_{n}(2 m)}{(2 m)^{k}} \sin 2 m \phi_{n}\right) \\
& -\frac{1}{r} \sum_{n=1}^{N}\left(\frac{A_{n}(2 m-1)}{(2 m)^{k}} \cos (2 m-1) \phi_{n}+\frac{B_{n}(2 m-1)}{(2 m)^{k}} \sin (2 m-1) \phi_{n}\right) \geq 0
\end{aligned}
$$

holds for all $m \in \mathbb{N}$ and, as previously,

$$
\begin{aligned}
\liminf _{m \rightarrow \infty} & \sum_{n=1}^{N}\left(a_{k}^{(n)} \cos 2 m \phi_{n}+b_{k}^{(n)} \sin 2 m \phi_{n}\right. \\
& \left.\left.-\frac{1}{r} a_{k}^{(n)} \cos (2 m-1) \phi_{n}-\frac{1}{r} b_{k}^{(n)} \sin (2 m-1) \phi_{n}\right)\right) \geq 0 .
\end{aligned}
$$

Since $\cos (2 m-1) \phi_{n}=\cos \phi_{n} \cos 2 m \phi_{n}+\sin \phi_{n} \sin 2 m \phi_{n}$ and $\sin (2 m-1) \phi_{n}=$ $\cos \phi_{n} \sin 2 m \phi_{n}-\sin \phi_{n} \cos 2 m \phi_{n}$, limit (3.2) is of the form as in Lemma 3.1. Therefore, 


$$
\begin{aligned}
\sum_{n=1}^{N} & \left(a_{k}^{(n)} \cos 2 m \phi_{n}+b_{k}^{(n)} \sin 2 m \phi_{n}\right. \\
& \left.-\frac{1}{r} a_{k}^{(n)} \cos (2 m-1) \phi_{n}-\frac{1}{r} b_{k}^{(n)} \sin (2 m-1) \phi_{n}\right)=0
\end{aligned}
$$

and, consequently, the equality

$$
\begin{aligned}
& \sum_{n=1}^{N}\left(a_{k}^{(n)} \cos 2 m \phi_{n}+b_{k}^{(n)} \sin 2 m \phi_{n}\right) \\
& \quad=\frac{1}{r} \sum_{n=1}^{N}\left(a_{k}^{(n)} \cos (2 m-1) \phi_{n}+b_{k}^{(n)} \sin (2 m-1) \phi_{n}\right)
\end{aligned}
$$

holds for all $m \in \mathbb{N}$. In the same manner we can show that

$$
\begin{aligned}
& \sum_{n=1}^{N}\left(a_{k}^{(n)} \cos 2 m \phi_{n}+b_{k}^{(n)} \sin 2 m \phi_{n}\right) \\
& \quad=r \sum_{n=1}^{N}\left(a_{k}^{(n)} \cos (2 m+1) \phi_{n}+b_{k}^{(n)} \sin (2 m+1) \phi_{n}\right) .
\end{aligned}
$$

Therefore, the sequence

$$
\left(r^{m} \sum_{n=1}^{N}\left(a_{k}^{(n)} \cos m \phi_{n}+b_{k}^{(n)} \sin m \phi_{n}\right)\right)_{m \in \mathbb{N}_{0}}
$$

is constant. In particular, $\sum_{n=1}^{N}\left(a_{k}^{(n)} \cos m \phi_{n}+b_{k}^{(n)} \sin m \phi_{n}\right)$ has a constant sign. Applying Lemma 3.1 once again, we conclude that (3.1) holds. The further reasoning is exactly the same as in the foregoing case.

Now we are in a position to prove one of our main results.

Theorem 3.3. Assume

$$
\begin{aligned}
& \mathcal{R}\left(a_{N}, \ldots, a_{0}\right)=\left\{\left(\lambda_{1}, l_{1}\right), \ldots,\left(\lambda_{p}, l_{p}\right),\left(\mu_{1}, k_{1}\right),\left(\overline{\mu_{1}}, k_{1}\right), \ldots,\left(\mu_{q}, k_{q}\right),\left(\overline{\mu_{q}}, k_{q}\right)\right\} . \\
& \text { If } \\
& \qquad\left|\lambda_{1}\right| \leq \cdots \leq\left|\lambda_{p}\right|<\left|\mu_{1}\right| \leq \cdots \leq\left|\mu_{q}\right|,
\end{aligned}
$$

then a continuous function $g: I \rightarrow I$ satisfies Eq. (1.1) if and only if it satisfies (1.4) with

$$
\mathcal{R}\left(b_{M}, \ldots, b_{0}\right)=\left\{\left(\lambda_{1}, l_{1}\right), \ldots,\left(\lambda_{p}, l_{p}\right)\right\} .
$$

Proof. Fix $x \in \mathbb{R}$ and define a sequence $\left(x_{m}\right)_{m \in \mathbb{N}_{0}}$ by putting

$$
x_{0}=x \quad \text { and } \quad x_{m}=g\left(x_{m-1}\right) \quad \text { for all } m \in \mathbb{N} .
$$


By Lemma 2.2 this sequence satisfies Eq. (1.3). Therefore, by Theorem 2.3, it is of the form

$x_{m}=F(m)+\sum_{n=1}^{q}\left|\mu_{n}\right|^{m}\left(A_{n}(m) \cos m \phi_{n}+B_{n}(m) \sin m \phi_{n}\right) \quad$ for all $m \in \mathbb{N}_{0} ;$

here $F$ stands for the part of the solution for which the roots $\lambda_{1}, \ldots, \lambda_{p}$ are responsible, $\phi_{n}$ is the principal argument of $\mu_{n}$ and $A_{n}, B_{n}$ are polynomials for $n=1, \ldots, q$. Applying Lemma 3.2 to $\left(x_{m}\right)_{m \in \mathbb{N}_{0}}$ one can eliminate the roots with modulus $\left|\mu_{q}\right|$. In each other step one can eliminate the non-real roots with the greatest modulus. This procedure yields $x_{m}=F(m)$ for all $m \in \mathbb{N}_{0}$. Hence, again by Theorem $2.3,\left(x_{m}\right)_{m \in \mathbb{N}_{0}}$ satisfies

$$
b_{M} x_{m+M}+\cdots+b_{1} x_{m+1}+b_{0} x_{m}=0
$$

and, consequently, (1.4) holds.

Corollary 3.4. Assume

$\mathcal{R}\left(a_{N}, \ldots, a_{0}\right)=\left\{\left(\lambda_{1}, l_{1}\right), \ldots,\left(\lambda_{p}, l_{p}\right),\left(\mu_{1}, k_{1}\right),\left(\overline{\mu_{1}}, k_{1}\right), \ldots,\left(\mu_{q}, k_{q}\right),\left(\overline{\mu_{q}}, k_{q}\right)\right\}$. If

$$
\left|\lambda_{1}\right| \geq \cdots \geq\left|\lambda_{p}\right|>\left|\mu_{1}\right| \geq \cdots \geq\left|\mu_{q}\right|
$$

then a continuous surjection $g: I \rightarrow I$ satisfies Eq. (1.1) if and only if it satisfies (1.4) with

$$
\mathcal{R}\left(b_{M}, \ldots, b_{0}\right)=\left\{\left(\lambda_{1}, l_{1}\right) \ldots,\left(\lambda_{p}, l_{p}\right)\right\} .
$$

Proof. By Lemma 2.1, case (3.5) may be reduced to case (3.3) by considering the dual equation. Thus, the assertion follows from Theorem 3.3.

Remark 3.5. It is well-known (see e.g. [21]) that if $I=\mathbb{R}$, then each continuous solution to (1.1) maps $\mathbb{R}$ onto $\mathbb{R}$. Hence the assumption of surjectivity in Corollary 3.4 is satisfied automatically in the case when $I=\mathbb{R}$.

Theorem 3.6. Assume

$$
\begin{aligned}
\mathcal{R}\left(a_{N}, \ldots, a_{0}\right)=\{ & (1,1),\left(\lambda_{1}, l_{1}\right) \ldots,\left(\lambda_{p}, l_{p}\right), \\
& \left.\left(\mu_{1}, k_{1}\right),\left(\overline{\mu_{1}}, k_{1}\right), \ldots,\left(\mu_{q}, k_{q}\right),\left(\overline{\mu_{q}}, k_{q}\right)\right\} .
\end{aligned}
$$

If

$$
\left|\lambda_{1}\right| \leq \cdots \leq\left|\lambda_{p}\right|<\left|\mu_{1}\right| \leq \cdots \leq\left|\mu_{q}\right|
$$

then a continuous function $g: I \rightarrow I$ satisfies Eq. (1.1) if and only if it satisfies (1.4) with

$$
\mathcal{R}\left(b_{M}, \ldots, b_{0}\right)=\left\{(1,1),\left(\lambda_{1}, l_{1}\right) \ldots,\left(\lambda_{p}, l_{p}\right)\right\}
$$


Proof. The proof is very similar to the proof of Theorem 3.3. Here the sequence $\left(x_{m}\right)_{m \in \mathbb{N}_{0}}$ has the form

$$
x_{m}=C+F(m)+\sum_{n=1}^{q}\left|\mu_{n}\right|^{m}\left(A_{n}(m) \cos m \phi_{n}+B_{n}(m) \sin m \phi_{n}\right)
$$

for all $m \in \mathbb{N}_{0}$, where $C$ is a constant. Since a constant has no effect on the monotonicity or the anti-monotonicity of a sequence, we can apply Lemma 3.2 and argue as before.

Corollary 3.7. Assume

$$
\begin{aligned}
\mathcal{R}\left(a_{N}, \ldots, a_{0}\right)=\{ & (1,1),\left(\lambda_{1}, l_{1}\right), \ldots,\left(\lambda_{p}, l_{p}\right), \\
& \left.\left(\mu_{1}, k_{1}\right),\left(\overline{\mu_{1}}, k_{1}\right), \ldots,\left(\mu_{q}, k_{q}\right),\left(\overline{\mu_{q}}, k_{q}\right)\right\} .
\end{aligned}
$$

If

$$
\left|\lambda_{1}\right| \geq \cdots \geq\left|\lambda_{p}\right|>\left|\mu_{1}\right| \geq \cdots \geq\left|\mu_{q}\right|
$$

then a continuous surjection $g: I \rightarrow I$ satisfies Eq. (1.1) if and only if it satisfies (1.4) with

$$
\mathcal{R}\left(b_{M}, \ldots, b_{0}\right)=\left\{(1,1),\left(\lambda_{1}, l_{1}\right), \ldots,\left(\lambda_{p}, l_{p}\right)\right\} .
$$

As an immediate corollary from the theorems above we obtain a result which for $I=\mathbb{R}$ was also proven in [16].

Corollary 3.8. If all the roots of the characteristic equation (1.2) are non-real, then Eq. (1.1) has no continuous solution $g: I \rightarrow I$.

\section{Eliminating roots of opposite sign}

Below we are going to prove that the order of the polynomial-like equation (1.1) can also be lowered when the minimal and the maximal (with respect to the absolute value) root of its characteristic equation are real and of opposite sign. However, it is necessary to distinguish two cases - when the unknown function monotonically increases and when it monotonically decreases.

Theorem 4.1. Assume

$$
\mathcal{R}\left(a_{N}, \ldots, a_{0}\right)=\left\{\left(r_{1}, k_{1}\right),\left(r_{2}, k_{2}\right),\left(\lambda_{1}, l_{1}\right), \ldots,\left(\lambda_{p}, l_{p}\right)\right\} .
$$

If

$$
\left|r_{1}\right|<\left|\lambda_{1}\right| \leq \cdots \leq\left|\lambda_{p}\right|<\left|r_{2}\right|
$$

and $r_{1}, r_{2}$ are real with $r_{1} r_{2}<0$, then a continuous increasing surjection $g: I \rightarrow I$ satisfies Eq. (1.1) if and only if it satisfies (1.4) with

$$
\mathcal{R}\left(b_{M}, \ldots, b_{0}\right)=\left\{\left(r_{j}, k_{j}\right),\left(\lambda_{1}, l_{1}\right), \ldots,\left(\lambda_{p}, l_{p}\right)\right\},
$$

where $j$ is the index of the positive number from $r_{1}$ and $r_{2}$. 
Proof. Fix $x \in \mathbb{R}$ and define a sequence $\left(x_{m}\right)_{m \in \mathbb{N}_{0}}$ by (3.4). By Lemma 2.2 this sequence satisfies Eq. (1.3). By replacing Eq. (1.1) with the dual equation, if necessary, we may assume that $r_{2}<0$. Since $g$ is increasing, $\left(x_{m}\right)_{m \in \mathbb{N}_{0}}$ is monotone and, by Theorem 2.3, of the form

$$
x_{m}=F(m)+A(m) r_{2}^{m} \quad \text { for all } m \in \mathbb{N}_{0},
$$

where $F$ represents the part of the solution to (1.3) for which the roots $r_{1}, \lambda_{1}, \ldots, \lambda_{p}$ are responsible and $A$ is a polynomial of degree at most $k_{2}-1$. Since

$$
x_{m}=m^{k_{2}-1} \cdot\left|r_{2}\right|^{m}\left(\frac{F(m)}{m^{k_{2}-1} \cdot\left|r_{2}\right|^{m}}+\frac{A(m)}{m^{k_{2}-1}}(-1)^{m}\right) \quad \text { for all } m \in \mathbb{N}
$$

and

$$
\lim _{m \rightarrow \infty} \frac{F(m)}{m^{k_{2}-1} \cdot\left|r_{2}\right|^{m}}=0, \quad \lim _{m \rightarrow \infty} \frac{A(m)}{m^{k_{2}-1}}=a_{k_{2}-1},
$$

where $a_{k_{2}-1}$ stands for the coefficient at $m^{k_{2}-1}$ in the polynomial $A$, the monotonicity of $\left(x_{m}\right)_{m \in \mathbb{N}_{0}}$ forces $a_{k_{2}-1}=0$. Continuing in this fashion, we eliminate all non-zero terms from $A$ and obtain $A \equiv 0$. It means that $x_{m}=$ $F(m)$ for all $m \in \mathbb{N}_{0}$ and, again by Theorem 2.3, it satisfies the relation $b_{M} x_{m+M}+\cdots+b_{1} x_{m+1}+b_{0} x_{m}=0$, which ends the proof.

Theorem 4.2. Assume

$$
\mathcal{R}\left(a_{N}, \ldots, a_{0}\right)=\left\{\left(r_{1}, k_{1}\right),\left(r_{2}, k_{2}\right),\left(\lambda_{1}, l_{1}\right), \ldots,\left(\lambda_{p}, l_{p}\right)\right\} .
$$

If

$$
\left|r_{1}\right|<\left|\lambda_{1}\right| \leq \cdots \leq\left|\lambda_{p}\right|<\left|r_{2}\right|
$$

and $r_{1}, r_{2}$ are real with $r_{1} r_{2}<0$, then a continuous decreasing surjection $g: I \rightarrow I$ satisfies Eq. (1.1) if and only if

(i) it satisfies (1.4) with

$$
\mathcal{R}\left(b_{M}, \ldots, b_{0}\right)=\left\{\left(r_{j}, k_{j}\right),\left(\lambda_{1}, l_{1}\right), \ldots,\left(\lambda_{p}, l_{p}\right)\right\},
$$

where $j$ is the index of the negative number from $r_{1}$ and $r_{2}$, in the case where $r_{1} \neq 1 \neq r_{2}$

or

(ii) it satisfies (1.4) with

$$
\mathcal{R}\left(b_{M}, \ldots, b_{0}\right)=\left\{(1,1),\left(r_{j}, k_{j}\right),\left(\lambda_{1}, l_{1}\right), \ldots,\left(\lambda_{p}, l_{p}\right)\right\},
$$

where $j$ is the index of the negative number from $r_{1}$ and $r_{2}$, in the case where the positive root from $r_{1}$ and $r_{2}$ equals 1 . 
Proof. Fix $x \in \mathbb{R}$ and define a sequence $\left(x_{m}\right)_{m \in \mathbb{N}_{0}}$ by (3.4). By Lemma 2.2 this sequence satisfies Eq. (1.3).

Firstly, we consider the case where $r_{1} \neq 1 \neq r_{2}$. By replacing Eq. (1.1) with the dual equation if necessary, we may assume that $r_{2}>0$. Since $g$ is decreasing, $\left(x_{m}\right)_{m \in \mathbb{N}_{0}}$ is anti-monotone and of the form

$$
x_{m}=F(m)+A(m) r_{2}^{m} \quad \text { for all } m \in \mathbb{N}_{0},
$$

where $F$ represents the part of the solution to (1.3) for which the roots $r_{1}, \lambda_{1}, \ldots, \lambda_{p}$ are responsible and $A$ is a polynomial of degree at most $k_{2}-1$. If it were $A \not \equiv 0$, we would have

$$
x_{m+1}-x_{m}=A(m) \cdot r_{2}^{m}\left(\frac{F(m+1)-F(m)}{A(m) \cdot r_{2}^{m}}+\frac{A(m+1)}{A(m)} r_{2}-1\right)
$$

for all $m \in \mathbb{N}_{0}$ and

$$
\lim _{m \rightarrow \infty}\left(\frac{F(m+1)-F(m)}{A(m) \cdot r_{2}^{m}}+\frac{A(m+1)}{A(m)} r_{2}-1\right)=r_{2}-1 \neq 0,
$$

which is a contradiction to the anti-monotonicity of $\left(x_{m}\right)_{m \in \mathbb{N}_{0}}$. Consequently, $x_{m}=F(m)$ for all $m \in \mathbb{N}_{0}$ and, similarly as in the previous proof, (1.4) is satisfied with $b_{j}$ 's as in assertion (i).

Secondly, we consider the case where either $r_{1}=1$ or $r_{2}=1$. By replacing Eq. (1.1) with the dual equation if necessary, we may assume that $r_{2}=1$. Again $\left(x_{m}\right)_{m \in \mathbb{N}_{0}}$ is anti-monotone and of the form

$$
x_{m}=F(m)+A(m) \text { for all } m \in \mathbb{N}_{0},
$$

where $F$ represents the part of the solution to (1.3) for which the roots $r_{1}, \lambda_{1}, \ldots, \lambda_{p}$ are responsible and $A$ is a polynomial of degree at most $k_{2}-1$. If the polynomial $A(m+1)-A(m)$ were non-zero, then denoting by $k$ its degree, we would have

$$
x_{m+1}-x_{m}=m^{k}\left(\frac{F(m+1)-F(m)}{m^{k}}+\frac{A(m+1)-A(m)}{m^{k}}\right)
$$

and

$$
\lim _{m \rightarrow \infty}\left(\frac{F(m+1)-F(m)}{m^{k}}+\frac{A(m+1)-A(m)}{m^{k}}\right)=a_{k} \neq 0,
$$

where $a_{k}$ stands for the coefficient at $m^{k}$ in $A(m+1)-A(m)$, which is a contradiction to the anti-monotonicity of $\left(x_{m}\right)_{m \in \mathbb{N}_{0}}$. Consequently, $A$ is constant and (1.4) holds with $b_{j}$ 's as in assertion (ii).

Reasoning in the same manner as in the proof of Theorem 3.6 and making use of the proof above, one can show that a single root 1 does not prevent the elimination of other roots. More precisely, we have the following result. 
Theorem 4.3. Assume

$$
\mathcal{R}\left(a_{N}, \ldots, a_{0}\right)=\left\{(1,1),\left(r_{1}, k_{1}\right),\left(r_{2}, k_{2}\right),\left(\lambda_{1}, l_{1}\right), \ldots,\left(\lambda_{p}, l_{p}\right)\right\} .
$$

If

$$
\left|r_{1}\right|<\left|\lambda_{1}\right| \leq \cdots \leq\left|\lambda_{p}\right|<\left|r_{2}\right|
$$

and $r_{1}, r_{2}$ are real with $r_{1} r_{2}<0$, then a continuous decreasing surjection $g: I \rightarrow I$ satisfies Eq. (1.1) if and only if it satisfies (1.4) with

$$
\mathcal{R}\left(b_{M}, \ldots, b_{0}\right)=\left\{(1,1),\left(r_{j}, k_{j}\right),\left(\lambda_{1}, l_{1}\right), \ldots,\left(\lambda_{p}, l_{p}\right)\right\},
$$

where $j$ is either the index of the negative number from $r_{1}$ and $r_{2}$, in the case of decreasing $g$, or the index of the positive number from $r_{1}$ and $r_{2}$, in the case of increasing $g$.

Remark 4.4. The assumption of surjectivity in Theorems 4.1, 4.2 and 4.3 is not essential when considering the dual equation is not necessary.

We finish this part of the present paper by observing that in each of the mentioned cases the order of Eq. (1.1) can be essentially lowered. The key property allowing us to use one of the results above is the monotonicity that is implied by the continuity of solutions to the considered equation.

\section{A generalized Zoltán Boros' problem}

In [3] the authors solved the original problem posed by Zoltán Boros (see [2]) during the 50th International Symposium on Functional Equations. Namely, we determined all continuous solutions to Eq. (1.5) for $n=3$. Let us recall this result.

Theorem 5.1. Assume $J \subset(0, \infty)$ to be an interval and let $f: J \rightarrow J$ be $a$ continuous solution to the equation $f^{3}(x)=[f(x)]^{3} / x^{2}$.

(i) If $J$ is bounded and $0 \notin \mathrm{cl} J$, then $f(x)=x$ for every $x \in J$.

(ii) If $J$ is bounded and $0 \in \mathrm{cl} J$, then there exists $c \in(0,1]$ such that

$$
f(x)=c x \quad \text { for every } x \in J .
$$

(iii) If $J$ is unbounded and $0 \notin \mathrm{cl} J$, then there exists $c \in[1, \infty)$ such that $(5.1)$ holds.

(iv) If $J=(0, \infty)$, then there exists $c \in(0, \infty)$ such that either $(5.1)$ holds or

$$
f(x)=\frac{c}{x^{2}} \quad \text { for every } x \in(0, \infty) .
$$

The next lemma gives a connection between the generalized Zoltán Boros' problem (1.5) and iterative equations of the form (1.1). Its proof is immediate and we omit it. 
Lemma 5.2. If $J \subset(0, \infty)$ is an interval and $f: J \rightarrow J$ is a solution to (1.5), then the formula $g=\log \circ f \circ \exp$ defines a function acting from $\log J$ into itself so that the equation

$$
g^{n}(x)=n g(x)-(n-1) x
$$

holds for every $x \in \log J$.

Conversely, if $I \subset \mathbb{R}$ is an interval and $g: I \rightarrow I$ is a solution to (5.2), then the formula $f=\exp \circ g \circ \log$ defines a function acting from $\exp I$ into itself so that (1.5) holds for every $x \in \exp I$.

Lemma 5.3. If a continuous and decreasing function $g: I \rightarrow I$ satisfies $E q$. (5.2), then $I=\mathbb{R}$. In particular, $g$ is surjective.

Proof. For an indirect proof suppose that $I \neq \mathbb{R}$. Put $a=\inf I$ and $b=\sup I$. If it were $a=-\infty$, then

$$
\infty=\lim _{x \rightarrow a}(n g(x)-(n-1) x)=\lim _{x \rightarrow a} g^{n}(x) \leq b<\infty,
$$

a contradiction. Similarly, if it were $b=\infty$, then

$$
-\infty<a \leq \lim _{x \rightarrow b} g^{n}(x)=\lim _{x \rightarrow b}(n g(x)-(n-1) x)=-\infty,
$$

a contradiction. Thus $a, b \in \mathbb{R}$. Put $c=\inf g(I)$. Since $g$ is decreasing, we have $a \leq c<b$. Further, we obtain

$$
c \leq \lim _{x \rightarrow b} g^{n}(x)=\lim _{x \rightarrow b}(n g(x)-(n-1) x)=n c-(n-1) b,
$$

which is an obvious contradiction to $c<b$.

For the part in particular see Remark 3.5.

The characteristic equation of (5.2) is of the form

$$
r^{n}=n r-n+1 \text {. }
$$

Since

$$
r^{n}-n r+n-1=(r-1)^{2}\left(\sum_{k=1}^{n-1} k r^{n-1-k}\right),
$$

it follows that 1 is a root of Eq. (5.3) of multiplicity 2. We will need two lemmas on the behaviour of the roots of this equation.

Lemma 5.4. (i) If $n \in 2 \mathbb{N}$, then Eq. (5.3) has no root in $\mathbb{R} \backslash\{1\}$.

(ii) If $n \in 2 \mathbb{N}+1$, then Eq. (5.3) has exactly one root $r_{0} \in \mathbb{R} \backslash\{1\}$. Moreover, $r_{0}$ is a single root and if $z \in \mathbb{C} \backslash \mathbb{R}$ is a root of $E q$. (5.3), then $|z|<-r_{0}$.

Proof. Define a function $f: \mathbb{R} \rightarrow \mathbb{R}$ by $f(x)=x^{n}-n x+n-1$. Then $f^{\prime}(x)=$ $n x^{n-1}-n$ and $f^{\prime \prime}(x)=n(n-1) x^{n-2}$ for every $x \in \mathbb{R}$.

(i) If $n$ is even, then the function $f$ is convex and has the global minimum at the point $x=1$. Hence $f(x)>f(1)=0$ for every $x \neq 1$. 
(ii) If $n$ is odd, then the function $f$ has a local maximum at the point $x=-1$ and a local minimum at the point $x=1$. Hence there exists a unique point $r_{0} \neq 1$ such that $f\left(r_{0}\right)=0$. Clearly, $r_{0}<-1$. Since $f^{\prime}\left(r_{0}\right) \neq 0$, we conclude that $r_{0}$ is a single root of Eq. (5.3).

Let $n=2 k+1 \geq 5$ with $k \in \mathbb{N}$. Consider a root $z \in \mathbb{C} \backslash \mathbb{R}$ of Eq. (5.3) and set all complex roots of this equation in the following sequence $\left(1,1, r_{0}, z, \bar{z}, w_{1}\right.$, $\left.\overline{w_{1}}, \ldots, w_{k-2}, \overline{w_{k-2}}\right)$; if $n=5$, then the sequence consists only of the first five elements. By Vieta's formulas we have $r_{0}\left|z w_{1} \cdots w_{k-2}\right|^{2}=-2 k$. This jointly with (5.3) yields

$$
r_{0}^{2 k}=2 k+1+\left|z w_{1} \ldots w_{k-2}\right|^{2} \quad \text { and } \quad z^{2 k}=2 k+1+r_{0} \bar{z}\left|w_{1} \ldots w_{k-2}\right|^{2} .
$$

Suppose that, contrary to our claim, $-r_{0} \leq|z|$. Then

$$
\begin{aligned}
|z|^{2 k} & =\left.\left.\left|2 k+1+r_{0} \bar{z}\right| w_{1} \cdots w_{k-2}\right|^{2}\left|<2 k+1-r_{0}\right| \bar{z}|\cdot| w_{1} \cdots w_{k-2}\right|^{2} \\
& \leq 2 k+1+\left|z w_{1} \cdots w_{k-2}\right|^{2}=r_{0}^{2 k}
\end{aligned}
$$

a contradiction.

Lemma 5.5. If $z \in \mathbb{C} \backslash \mathbb{R}$ is a root of Eq. (5.3), then $|z|>1$.

Proof. Let $z=|z|(\cos \varphi+\mathrm{i} \sin \varphi)$. Since $z \notin \mathbb{R}$, we have $|z|>0$ and $\sin \varphi \neq 0$. By (5.3) we conclude that $|z|^{n} \sin n \varphi=|z| n \sin \varphi$, and hence that

$$
|z|=\sqrt[n-1]{\frac{n \sin \varphi}{\sin n \varphi}} .
$$

Now it is enough to show that

$$
|\sin n \varphi|<n|\sin \varphi| \text {. }
$$

We prove, by induction, that (5.4) holds for all integers $n \geq 2$ and reals $\varphi$ such that $\sin \varphi \neq 0$ or, equivalently, $|\cos \varphi|<1$.

Clearly, $|\sin 2 \varphi|=2|\sin \varphi \cos \varphi|<2|\sin \varphi|$. Consider $n \geq 2$ and assume that (5.4) holds for every $\varphi$ with $\sin \varphi \neq 0$. Then

$$
\begin{aligned}
|\sin (n+1) \varphi| & =|\sin (n \varphi+\varphi)| \leq|\sin n \varphi \cos \varphi+\cos n \varphi \sin \varphi| \\
& \leq|\sin n \varphi \cos \varphi|+|\cos n \varphi \sin \varphi|<|\sin n \varphi|+|\sin \varphi| \\
& <n|\sin \varphi|+|\sin \varphi|=(n+1)|\sin \varphi|,
\end{aligned}
$$

which completes the proof.

Now we can formulate and prove the main result of this section.

Theorem 5.6. If a continuous function $g: I \rightarrow I$ satisfies $E q$. (5.2), then either it satisfies the equation

$$
g^{2}(x)-2 g(x)+x=0
$$

in the case where $g$ is increasing or it satisfies the equation

$$
g^{2}(x)-\left(r_{0}+1\right) g(x)+r_{0} x=0,
$$


where $r_{0}$ stands for the negative root of the polynomial $r^{n}-n r+(n-1)$, in the case where $g$ is decreasing.

Proof. If $n$ is an even number, then by Lemma 5.4 the only real root of (5.3) is 1 (whose multiplicity equals 2) and by Lemma 5.5 all non-real roots have modulus greater than 1 . Theorem 3.3 yields that (5.5) holds. In this case Eq. (5.2) directly forces $g$ to be increasing.

If $n$ is an odd number, then by Lemma 5.4, Eq. (5.3) has two real roots: 1 of multiplicity 2 and a single negative root $r_{0}$. Assuming $g$ to be increasing and using Theorem 4.1 (and also Remark 4.4) we can eliminate $r_{0}$ from the characteristic Eq. (5.3). Furthermore, using Theorem 3.3, we can eliminate all non-real roots. Consequently, (5.5) holds. If $g$ is decreasing, then by Lemma 5.3 it is surjective. Now using Theorems 4.2 and 3.6, we eliminate the single root 1 and all non-real roots from (5.3). Finally, we conclude that (5.6) holds.

The next theorem extends Theorem 5.1 and solves the problem of Zoltán Boros in the general case.

Theorem 5.7. Assume $J \subset(0, \infty)$ to be an interval and let $f: J \rightarrow J$ be $a$ continuous solution to Eq. (1.5).

(i) If $J$ is bounded and $0 \notin \mathrm{cl} J$, then $f(x)=x$ for every $x \in J$.

(ii) If $J$ is bounded and $0 \in \mathrm{cl} J$, then there exists $c \in(0,1]$ such that

$$
f(x)=c x \quad \text { for every } x \in J .
$$

(iii) If $J$ is unbounded and $0 \notin \mathrm{cl} J$, then there exists $c \in[1, \infty)$ such that $(5.7)$ holds.

(iv) If $J=(0, \infty)$ and $n$ is even, then there exists $c \in(0, \infty)$ such that $(5.7)$ holds.

(v) If $J=(0, \infty)$ and $n$ is odd, then there exists $c \in(0, \infty)$ such that either (5.7) holds or

$$
f(x)=c x^{r_{0}} \quad \text { for every } x \in(0, \infty),
$$

where $r_{0}$ is the negative root of (5.3).

Proof. By substituting $g=\log \circ f \circ \exp$ and using Lemma 5.2 we can reduce Eq. (1.5) to (5.2), where $g$ acts from $I=\log J$ into $I$.

In assertions (i)-(iii), by Lemma 5.3, $g$ is increasing. By Theorem 5.6, $g$ satisfies (5.5). Since $r^{2}-2 r+1$ divides $r^{3}-3 r+2$, the function $g$ satisfies (5.2) with $n=3$. Consequently, $f$ satisfies (1.5) with $n=3$. Therefore, by Theorem 5.1, $f(x)=c x$ for some positive $c$. Since $f$ ranges in $J$, it forces $c=1$ in case (i), $c \in(0,1]$ in case (ii) and $c \in[1, \infty)$ in case (iii). One may easily verify that each of these functions solves (1.5).

In assertion (iv), by Eq. (5.2) $g$ is increasing and by Theorem 5.6 it satisfies (5.5). The same reasoning as above shows that $f$ is given by (5.7). One may easily verify that such an $f$ solves (1.5) for arbitrary positive $c$. 
In assertion (v) the increasing solutions are obtained exactly in the same manner as in the preceding item. Assume $g$ to be decreasing. Then, by Theorem 5.6, it satisfies (5.6). According to [13], we conclude that $g(x)=r_{0} x+c_{0}$ for some constant $c_{0}$ and, consequently, $f(x)=c x^{r_{0}}$ for some positive $c$. Again such an $f$ solves (1.5) for arbitrary $c \in(0, \infty)$.

\section{Questions and remarks}

By $[9,13]$ if the characteristic equation (1.2) has at least two distinct positive roots or two real opposite roots, then the solution to (1.1) is not unique (it depends on an arbitrary function). Results from Sects. 3 and 4 allow us to determine solutions to (1.1), provided that they are unique, in many cases. Of particular interest is the situation, still unsolved in full generality, when the characteristic equation has non-real roots. In view of our results it is natural to ask the following question.

Problem 6.1. Set $\varrho=\max \{|r|: r$ is a root of (1.2) $\}$. Is it possible to eliminate non-real roots with modulus $\varrho$ from the characteristic equation (1.2) when this equation has also a real root with modulus $\varrho$ ?

It seems that non-real roots of the characteristic equation do not affect solutions to polynomial-like iterative equations in any case, but this problem is still open (see [21]).

\section{Acknowledgements}

This research was supported by the University of Silesia Mathematics Department (Iterative Functional Equations and Real Analysis program).

Open Access. This article is distributed under the terms of the Creative Commons Attribution 4.0 International License (http://creativecommons.org/licenses/by/4.0/), which permits unrestricted use, distribution, and reproduction in any medium, provided you give appropriate credit to the original author(s) and the source, provide a link to the Creative Commons license, and indicate if changes were made.

\section{References}

[1] Baron, K., Jarczyk, W.: Recent results on functional equations in a single variable, perspectives and open problems. Aequ. Math. 61, 1-48 (2001)

[2] Boros, Z.: Talk given during the 50th international symposium on functional equations. Aequ. Math. 86, 293 (2013)

[3] Draga, S., Morawiec, J.: On a Zoltán Boros' problem connected with polynomial-like iterative equations. Nonlinear Anal. Real 26, 56-63 (2015)

[4] Jarczyk, W.: On an equation of linear iteration. Aequ. Math. 51, 303-310 (1996) 
[5] Jerri, A.J.: Linear Difference Equations with Discrete Transform Methods. Springer, Berlin (1996)

[6] Li, L., Zhang, W.: Continuously decreasing solutions for polynomial-like iterative equations. Sci. China Math. 56, 1051-1058 (2013)

[7] Matkowski, J.: Remark done during the twenty-sixth international symposium on functional equations. Aequ. Math. 37, 119 (1989)

[8] Matkowski, J., Zhang, W.: Method of characteristic for functional equations in polynomial form. Acta Math. Sin. 13, 421-432 (1997)

[9] Matkowski, J., Zhang, W.: Characteristic analysis for a polynomial-like iterative equation. Chin. Sci. Bull. 43, 192-196 (1998)

[10] Morawiec, J.: On a functional equation involving iterates and powers. Adv. Differ. Equ. 2014, 271 (2014)

[11] Mukherjea, A., Ratti, J.S.: On a functional equation involving iterates of a bijection on the unit interval. Nonlinear Anal. 7, 899-908 (1983)

[12] Mukherjea, A., Ratti, J.S.: On a functional equation involving iterates of a bijection on the unit interval, II. Nonlinear Anal. 31, 459-464 (1998)

[13] Nabeya, S.: On the functional equation $f(p+q x+r f(x))=a+b x+c f(x)$. Aequ. Math. 11, 199-211 (1974)

[14] Ratti, J.S., Lin, Y.F.: A functional equation involving $f$ and $f^{-1}$. Colloq. Math. 60/61, 519-523 (1990)

[15] Tabor, J., Tabor, J.: On a linear iterative equation. Results Math. 27, 412-421 (1995)

[16] Yang, D., Zhang, W.: Characteristic solutions of polynomial-like iterative equations. Aequ. Math. 67, 80-105 (2004)

[17] Zhang, W., Baker, A.J.: Continuous solutions of a polynomial-like iterative equation with variable coefficients. Ann. Pol. Math. 73, 29-36 (2000)

[18] Zhang, P., Gong, X.: Continuous solutions of 3-order iterative equation of linear dependence. Adv. Differ. Equ. 2014, 318 (2014)

[19] Zhang, W., Xu, B., Zhang, W.: Global solutions for leading coefficient problem of polynomial-like iterative equations. Results Math. 63, 79-93 (2013)

[20] Zhang, J., Yang, L., Zhang, W.: Some advances on functional equations. Adv. Math. (China) 24, 385-405 (1995)

[21] Zhang, W., Zhang, W.: On continuous solutions of $n$-th order polynomial-like iterative equations. Publ. Math. Debr. 76, 117-134 (2010)

\author{
Szymon Draga \\ Institute of Mathematics \\ University of Silesia \\ Bankowa 14 \\ 40-007 Katowice \\ Poland \\ e-mail: szymon.draga@gmail.com \\ Janusz Morawiec \\ Institute of Mathematics \\ University of Silesia \\ Bankowa 14 \\ 40-007 Katowice \\ Poland \\ e-mail: janusz.morawiec@us.edu.pl
}

Received: December 10, 2015 